\title{
Marginalien Konrad Gessners als historische Quelle
}

\author{
Huldrych M. Koelbing in gemeinsamer Gessner-Freundschaft zum \\ 70. Geburtstag
}

Urs B. Leu

\section{SUMMARY}

Conrad Gessner (1516-1565) possessed an impressive library of hundreds of books. A lot of these books distinguish themselves by their hand-written notes made by Gessner. Nobody took much notice of them until a short time ago. From his own copy of his Bibliotheca universalis it becomes clear that his marginal notes could be of interest and of importance for several aspects of the history of the early modern period.

\section{ZuSAMMENFASSUNG}

Konrad Gessner (1516-1565) besass eine stattliche, mehrhundertbändige Privatbibliothek. ${ }^{1}$ Viele seiner Bücher zeichnen sich dadurch aus, dass sie geradezu übersät sind mit handschriftlichen Annotationen (Marginalien) Gessners. Dieser enorme handschriftliche Fundus ist von der Gessnerforschung bis vor kurzem kaum zur Kenntnis genommen worden. Am Beispiel von Gessners Handexemplar der von ihm selber verfassten Bibliotheca universalis wird deutlich, dass seine Marginalien für gewisse Aspekte der Geschichte der frühen Neuzeit von Interesse, ja vielleicht sogar von Bedeutung sein können.

Konrad Gessners erstes Werk, das ihn weit über die Grenzen der Schweiz hinaus bekannt werden liess, war die 1545 bei Christoph Froschauer dem Älteren in Zürich erschienene Bibliotheca universalis. ${ }^{2}$ Der damals $29 \mathrm{jährige}$ Mediziner veröffentlichte damit die erste gedruckte, internationale Bibliographie. Es fanden alle ihm bekannten gedruckten und ungedruckten Werke Aufnahme, die in einer der gelehrten drei Sprachen des Altertums und des Humanismus, nämlich auf Hebräisch, Griechisch oder Lateinisch, abgefasst 
worden waren. Zum Teil wurden aber auch ursprünglich volkssprachliche Werke berücksichtigt, besonders diejenigen von bedeutenden Reformatoren, wobei die Titelaufnahme in lateinischer Übersetzung erfolgte. Insgesamt gelang es Gessner, wahrscheinlich einige zehntausend Titel zu verzeichnen. ${ }^{3}$ Grundlage dazu bildeten bereits bestehende Schriftsteller- und Werkverzeichnisse unterschiedlicher Provenienz ${ }^{4}$ sowie Kontakte zu Bibliophilen wie auch zahlreiche Besuche in den verschiedensten mitteleuropäischen Bibliotheken.

Auch nach diesem glänzenden Wurf sammelte Gessner zeit seines Lebens alle nur irgendwie greifbaren bibliographischen Informationen und kaufte sich auch selber viele Bücher. Innerhalb der folgenden beiden Dezennien publizierte er mindestens sechs weitere anspruchsvolle bibliographische Arbeiten: 1551 schickte er dem ersten Band der Historia animalium ein Verzeichnis aller ihm bekannten Tierschriftsteller voraus, 1552 folgte in der Praefatio rei herbariae zur lateinischen Ausgabe von Hieronymus Bocks De stirpium facultatibus eine botanisch orientierte Bibliographie und bereits wieder 1555 verfasste er ein entsprechendes Verzeichnis für die Chirurgie, das im von ihm selber herausgegebenen Sammelband Chirurgia veröffentlicht wurde. 1558 arbeitete er für den vierten Band der Historia animalium ein Schriftstellerverzeichnis zum Thema Wassertiere aus, 1561 listete er in den Annotationes in Dioscorides alle Kommentare zu Pedacius Dioscorides Anazarbus auf und 1562 schliesslich erschien die vielgerühmte Galenbibliographie zur grossen, von Froben in Basel gedruckten Galenausgabe.

Die Bibliotheca universalis entsprach einem gewissen Bedürfnis der Zeit, die immer unübersehbarer werdende Fülle an gedruckten Publikationen in einem übersichtlichen Verzeichnis zu ordnen, und konnte sich daher eines breiten Leserkreises erfreuen. Auch der gelehrte Zürcher Antistes und Mentor Gessners, Heinrich Bullinger (1504-1575), benutzte die Bibliotheca universalis zu persönlichen Studienzwecken ${ }^{5}$ und schenkte dem St.Galler Freund und Reformator Joachim Vadian (1484-1551) ein Exemplar mit handschriftlicher Widmung. ${ }^{6}$ Der Zürcher Hebräischprofessor und Bibliothekar am Grossmünsterstift, Konrad Pellikan (1478-1556), verwendete die Bibliotheca universalis als eigentlichen Bibliothekskatalog. ${ }^{7}$ Auch in der Humanistenstadt Basel stand Gessners Bibliographie in hohem Ansehen. Fast zwanzig Jahre nach Erscheinen galt sie immer noch als das massgebliche Schriftstellerlexikon, weshalb der Basler Schulmeister Jacob Härtlein (1536-1564) zu Beginn einer von Hieronymus Froben (1501-1563) und Nicolaus Episcopius (1501-1564) besorgten Ausgabe des griechischen Kir- 


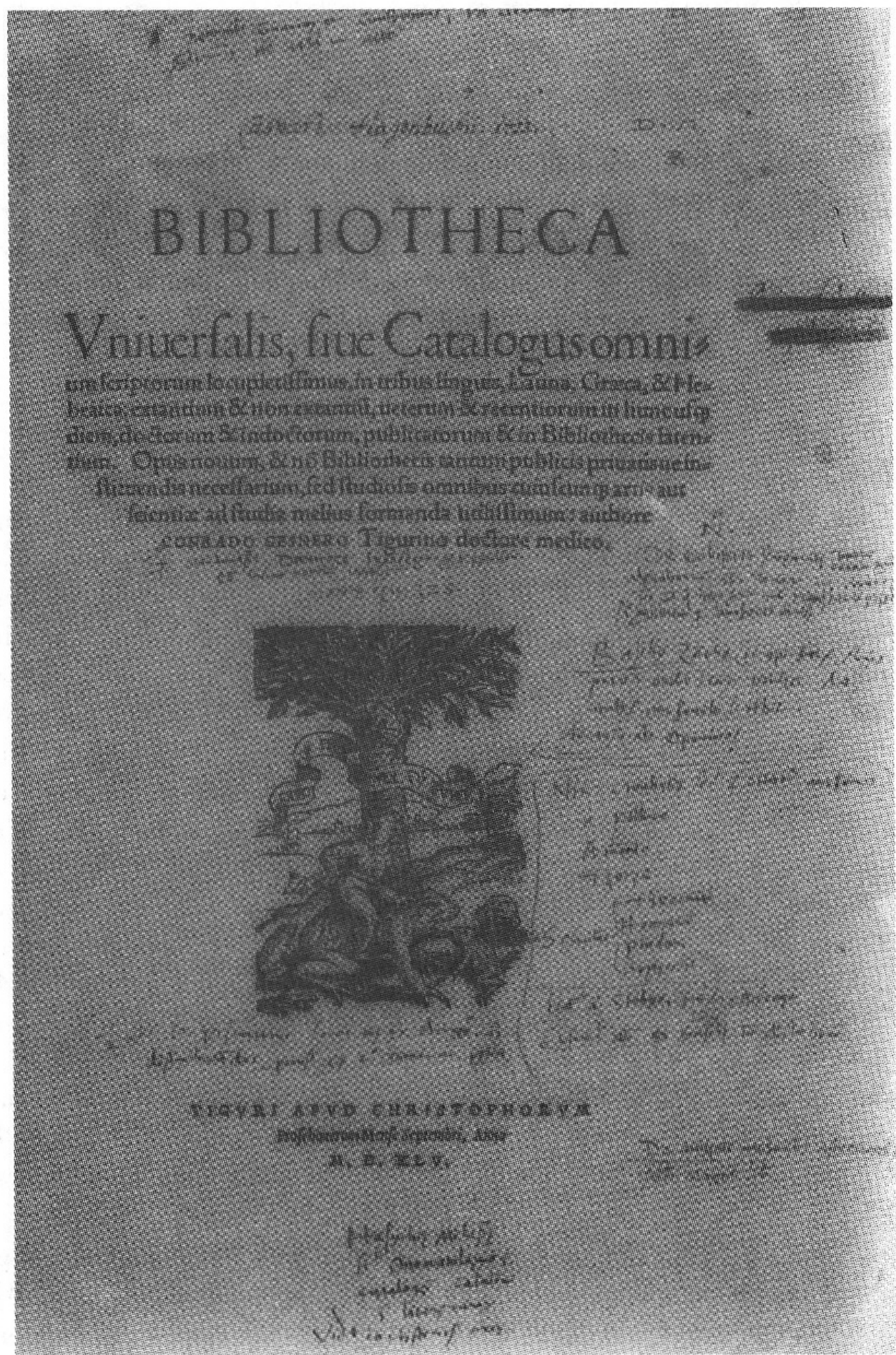

Konrad Gessners Handexemplar der «Bibliotheca universalis»(Zentralbibliothek Zürich). 
chenvaters Cyrill v. Alexandrien (5. Jh.) bemerkte, dass wer mehr über Cyrill und seine Schriften wissen wolle, bei Gennadius und vor allem in der Bibliotheca universalis nachschlagen solle, wo er viel Lesens- und Wissenswertes finden werde. ${ }^{8} \mathrm{Gessner}$, wohl nicht zuletzt erfreut und motiviert durch das breite Echo, annotierte in seinem eigenen Exemplar der Bibliotheca universalis mit Akribie Korrekturen, Neuerscheinungen, inhaltliche Ergänzungen u.v. m., wohl nicht zuletzt im Hinblick auf eine Neuauflage seiner epochalen Universalbibliographie.

\section{Konrad Gessners Bücher und Johann Caspar Hagenbuch}

Gessner vermachte seine Bücher und Manuskripte seinem Nachfolger im Amt, dem späteren Stadtarzt Kaspar Wolf (1532-1601). Wolf bezeugt dies selber in seinem gedruckten Versprechen, den botanischen Nachlass Gessners sachgerecht zu verwalten und herauszugeben: «Igitur eo mortuo, cum haec eius libraria suppellex omnis eo pacto ad me translata esset, et illud chaos ego utcunque digesissem, ... ${ }^{9}{ }^{9}$ Gessners Freund, der Theologe Josias Simmler (1530-1576), bestätigt dies und ruft Wolf in der Vorrede seiner Gessner-Biographie zu: «Tuque praesertim ornatissime Vuolphi, qui nunc omnem illius bibliothecam possides, optime testari potes, quanta fuerit virtute, doctrina et diligentia praeditus Gesnerus noster, quando tibi quotidie libros et scripta eius evoluenti, innumera harum rerum vestigia occurrunt..s ${ }^{10}$ Wolf hielt Gessners Bücher- und Manuskriptenschatz nicht sehr lange zusammen, sondern verkaufte einiges davon an interessierte Gelehrte wie etwa den Nürnberger Arzt und Botaniker Joachim Camerarius (15431598) ${ }^{11}$ oder den Basler Mediziner Felix Platter (1536-1614). ${ }^{12}$ In wessen Hände er Gessners Handexemplar der Bibliotheca universalis übergab, ist nicht bekannt. Sicher ist aber, dass der berühmte Zürcher Theologe und Altertumsforscher Johann Caspar Hagenbuch (1700-1763) ${ }^{13}$ im Jahr 1723 in den Besitz des Buches gelangte. Er notierte nämlich handschriftlich auf das Titelblatt: «Caspari Hagenbuchii. 1723.»

Hagenbuch scheint eine grosse Vorliebe für Werke aus der ehemaligen Gessnerschen Privatbibliothek gehabt zu haben, denn in den Jahren 1720 bis 1730 erwarb er mindestens 35 Schriften in 22 Bänden aus Gessners Besitz. Vor allem während des Jahres 1723 gelang es ihm, einige Gessneriana aufzuspüren. ${ }^{14}$ Hagenbuch schätzte diese Zimelien seiner Büchersammlung sehr und schenkte den von Gessner angebrachten Marginalien als erster 
bewusst Beachtung. Dies kommt beispielsweise in seinem handschriftlich verfassten Schriftstellerlexikon Bibliotheca auctorum alphabetica zum Ausdruck, wo er zu Gessners Bibliotheca universalis festhält:

«Per integrum hoc opus suum Gesnerus in exemplari, quod possideo, quoque ipse usus fuerat, singulis fere paginis propria manu plurima adscripsit tum edita corrigens, tum multa nova addens. ${ }^{15}$

Ein Buch Gessners, das Hagenbuch seit 1722 in seiner Bibliothek stehen hatte, ging geradezu in die Geschichte der Klassischen Philologie ein, nämlich Gessners ebenfalls reich annotiertes Handexemplar der von ihm besorgten Ausgabe des antiken Tierschriftstellers Aelian (ca. 170-240 n. Chr.), die 1556 in Zürich erschienen war. ${ }^{16}$ Hagenbuch schrieb zahlreiche Marginalien Gessners daraus ab und liess sie dem Leidener Juristen, Philologen und Bibliothekar Abraham Gronovius (1694-1775) ${ }^{17}$ zu Handen seiner Arbeiten an einer neuen Aelianausgabe zukommen. Gronovius wusste dieses grosszügige Entgegenkommen Hagenbuchs sehr zu schätzen, setzte u. a. Gessners Namen als Bearbeiter mit auf das Titelblatt und dankte im Vorwort der Erstausgabe seiner Edition von 1731 mit folgenden Worten:

«Insuper contexui subjunxi integras notas Gesneri, Schefferi, Fabri, Konigii, Kuhnii, et Perizonii, quibus etiam accedunt curae secundae Conradi Gesneri, quas debere me agnosco humanitati Reverendi admodum, et doctissimi Viri, Joannis Caspari Hagenbuchi, qui singula, quae alter ille aevi sui Plinius in exemplari Operum Aeliani a se edito adnotaverat, accurate ad me transmisit. ${ }^{18}$

Im gleichen Jahr noch berichteten die Acta eruditorum ${ }^{19}$ über diesen Handel. Auch in den folgenden Jahren und Jahrzehnten erinnerte man sich immer wieder an Hagenbuchs Uneigennützigkeit und Gessners zeitüberdauernde Leistungen. Sogar im knapp gehaltenen Nachruf für Hagenbuch in den Monatlichen Nachrichten einicher Merkwürdigkeiten, in Zürich gesammelt, und herausgegeben vom Jahre MDCCLXIII wird festgehalten:

«Nicht weniger unterhielte er einen merkwürdigen Brief-Wechsel mit dem gelehrten Bibliothecarius in Leiden, Abraham Gronovius, welchem er in demselbigen Jahre Conrad Gessners, in Handschrift hinterlassene Anmerkungen über den Aelian, nebst seinen eigenen überschikte; dass Gronovius sich derselben bey der doppelten Ausgabe dieses Schriftstellers nützlich bedienet habe, wird in den Actis Erud. 1731. p.499. und anderstwo gemeldt.»

Nach Hagenbuchs Tod am 6. Juni 1763 ging seine über dreitausendbändige Privatbibliothek an seinen Sohn, der aber nichts damit anzufangen wusste. 


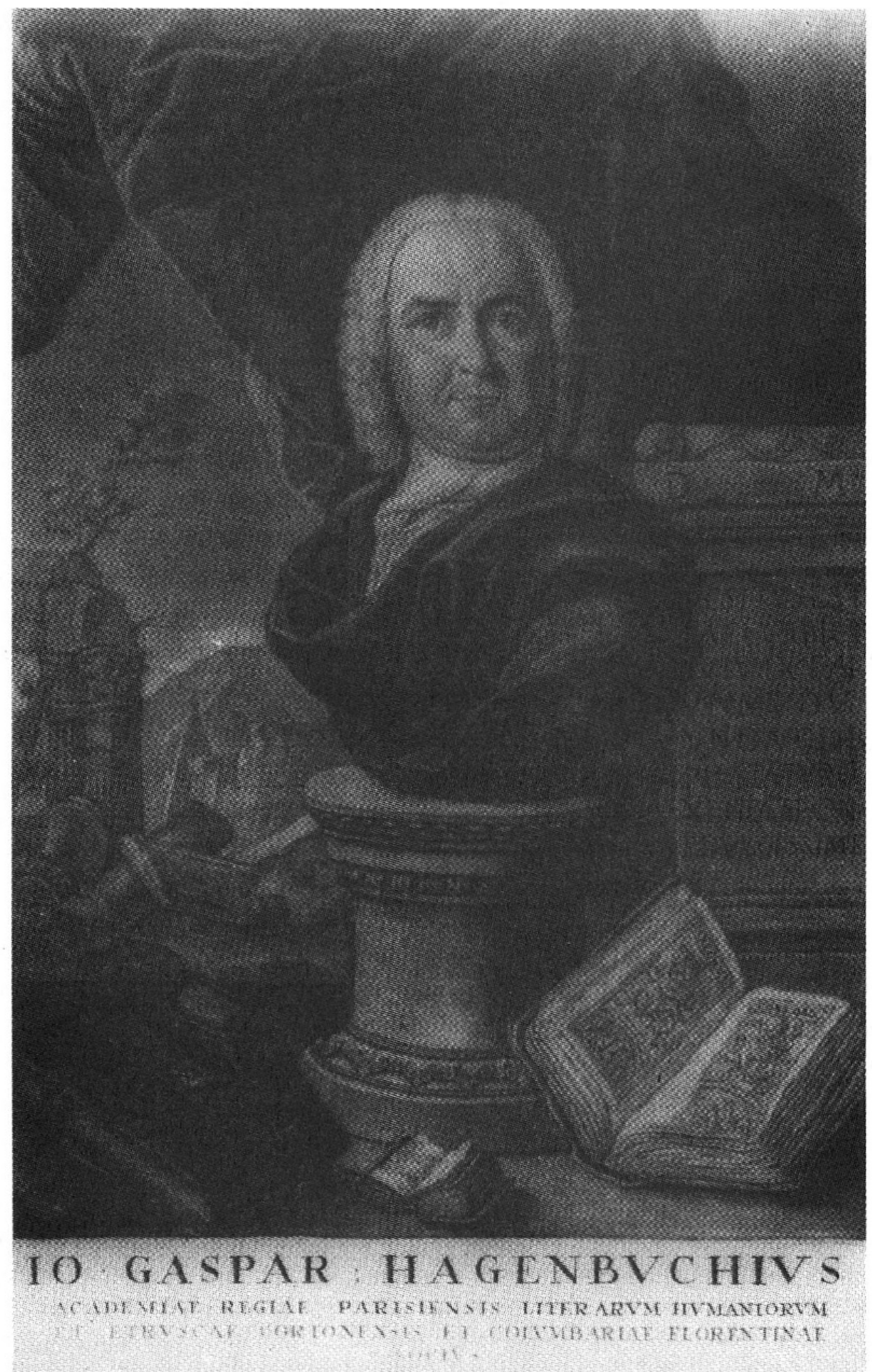

Johann Caspar Hagenbuch (1700-1763): Mezzotinto nach einem Gemälde von Johann Caspar Füssli d. A., erschienen in Zürich 1753 (Zentralbibliothek Zürich). 
Dieser willigte schliesslich am 25. September 1764 ein, den Bücherschatz seiner bildungshungrigen Schwester Susanna (1732-1805) als Bräutigamsgeschenk für ihren Gatten, den Zürcher Kanonikus Johann Jakob Steinbrüchel $(1729-1796)^{20}$, zu verkaufen. Eigenartigerweise ist Gessners Handexemplar aber im handschriftlichen Katalog der Hagenbuch-Bibliothek aus dem Jahr 1764 nicht mehr aufgeführt. ${ }^{21}$ Es taucht ein letztes Mal im entsprechenden Inventar aus dem Jahr 1758 auf, das Hagenbuch noch selber angelegt hatte..$^{22}$ Hat Hagenbuch vielleicht Gessners Handexemplar der Bibliotheca universalis zu seinen Lebzeiten jemandem ausgeliehen? Der im Jahre 1809 erschienene, gedruckte Katalog der Zürcher Stadtbibliothek verzeichnet Gessners Handexemplar der Bibliotheca universalis jedenfalls wieder unter der Signatur «Sp» ${ }^{23}$, welche Turicensia umfasst. Dass das Werk nicht in einer der typischen Hagenbuch-Signaturen XIX, XX, XXI oder XXII aufgestellt worden war, ist ein Hinweis mehr dafür, dass es unabhängig vom Hagenbuch-Nachlass in die Stadtbibliothek gelangt ist. Etwa in der Mitte des letzten Jahrhunderts wurde das Werk in die weiter gefasste Signatur «PA» umgestellt, die Helvetica enthält, womit es wahrscheinlich bis zu seiner Wiederentdeckung in den sechziger Jahren dieses Jahrhunderts durch den Bibliothekar und Gessnerforscher Dr. Rudolph Steiger (18961978) ${ }^{24}$ in Vergessenheit geriet.

Gessners Handexemplar der Bibliotheca universalis verdient unter den Büchern aus dem ehemaligen Besitz Konrad Gessners besondere Beachtung, weil es überdurchschnittlich viele Marginalien Gessners aufweist und diese nicht nur Korrekturen und Nachträge, sondern darüber hinaus viele historisch interessante Bemerkungen enthalten. Von den annotierten Addenda und Corrigenda sind die meisten in die von Josias Simmler besorgte Epitome Bibliothecae Conradi Gesneri von 1555 oder in die ebenfalls von Simmler bearbeitete Bibliotheca instituta ... in Epitomen redacta von 1574 eingeflossen. Hingegen diejenigen Randnotizen, die für die Geistes- und Gelehrtengeschichte des 16. Jahrhunderts Bedeutung haben, sind bis jetzt kaum beachtet, geschweige denn ausgewertet worden.

Die ältesten von Gessner datierten handschriftlichen Zusätze stammen aus dem Jahr $1548^{25}$, die jüngsten von $1564 . .^{26}$ Das schliesst selbstredend nicht aus, dass er auch vorher sowie nachher gewisse Einträge vorgenommen hat. Die zeitgeschichtlichen Mitteilungen in Gessners Marginalien sind höchst unterschiedlicher Natur. Er notierte, was ihm einfiel, was ihm wichtig schien, was ihn selber innerlich bewog, jenseits jeglicher Systematik. 


\section{Über Bücher und ihre Preise}

Als lebenslänglich von Finanznöten gedrückter Bibliophiler und Bibliograph interessierte sich Gessner nicht nur für die Druckwerke an sich, sondern auch für ihre Preise. An zwei Stellen annotierte er daher die entsprechende Kaufsumme: Einerseits zur Gesamtausgabe der Werke des Kirchenvaters Augustin und anderseits zum Tierbuch des Engländers Edward Wotton. ${ }^{27}$ Während der Eintrag zum letzteren nicht besonders aussagekräftig ist, enthält derjenige zu Augustins Werken eine gewisse Brisanz. Gessner vergleicht den Preis der von Froben in Basel verlegten Augustinausgabe von 1529 mit derjenigen, die 1541 in Paris bei Yolande Bonhomme erschienen ist :

«Den Pariser umb 12 Gulden/ und den Frobenianisch ietz/auch, for[her] gab man ihn umb/ $15 .{ }^{28}$

Eventuell nimmt Gessner hier bereits Bezug auf die entsprechende Basler Neuauflage, denn das Haus Froben legte die Augustin-Gesamtausgabe 1543 ein zweites Mal auf, wobei die Produktionskosten sicher tiefer gehalten werden konnten als bei der Erstausgabe, da gewisse wissenschaftliche und wahrscheinlich auch drucktechnische Vorarbeiten nicht mehr zu bewältigen waren. Vielleicht hat Froben den Preis auch deshalb dem Pariser Niveau angepasst, um im Preiskampf nicht zu unterliegen und ausgebootet zu werden. Diese von Gessner festgehaltene Reduktion des Buchpreises ist von besonderem Interesse für die Wirtschaftsgeschichte der frühen Neuzeit, weil die Quellen sonst grundsätzlich von einem Ansteigen der Preise während des ganzen 16. Jh. sprechen. ${ }^{29}$ Im deutschsprachigen Raum scheint sich das Preisniveau etwa ab dem ersten Viertel des 16. Jh. bis zum Vorabend des dreissigjährigen Krieges, zwar mit zeitweise massiven Schwankungen, aber trotzdem unablässig erhöht zu haben. ${ }^{30}$

Dieser neue, von Gessner angeführte Preis der Frobener Ausgabe entsprach wohl einem der tiefsten während der ersten Hälfte des 16. Jh., denn der Zürcher Reformator Heinrich Bullinger (1504-1574) beispielsweise musste für die gleiche Frobenausgabe im Jahr 1534 immerhin noch 46 Pfund berappen $^{31}$, was ungefähr 23 Gulden entsprach. ${ }^{32} Z_{w a r}$ muss bei diesem Vergleich berücksichtigt werden, dass Gessner sehr wahrscheinlich die ungebundene und damals handelsübliche Ausgabe im Auge hatte und dass Bullinger wohl für die bereits gebundenen acht Bände soviel bezahlt hat. Das Binden eines Foliobandes dürfte ungefähr etwas mehr als einen Gulden 
gekostet haben. ${ }^{33}$ Bullinger hätte daher 1534 für den ungebundenen Augustin um die 15 Gulden bezahlen müssen, was dem von Gessner erwähnten früheren Frobenschen Preisniveau entsprochen hätte. Für Gessner waren selbstredend auch ein Dutzend Gulden noch viel Geld, zumal er bis zur Erlangung einer Chorherrenstelle im September $1558 \mathrm{im}$ Jahr lediglich 160 Pfund bzw. 80 Gulden verdiente. ${ }^{34}$ Zwölf Gulden entsprachen etwa einem Siebentel seines Jahreseinkommens! Kein Wunder arbeitete er u. a. nebenamtlich für die Druckerei Froschauer, um sein Gehalt etwas aufzubessern. ${ }^{35}$

\section{Zeitgeschichtliche Mitteilungen}

Aber nicht nur den Büchern, sondern auch ihren Autoren schenkte Gessner zeit seines Lebens grosse Beachtung, suchte zu vielen den persönlichen Kontakt, hielt sich selber wo immer möglich auf dem laufenden und annotierte dies und das in seinem persönlichen Exemplar der Bibliotheca universalis. Nebst den Sterbedaten gewisser Gelehrter, die eigenartigerweise häufig Fehler von bis zu einem Jahr aufweisen ${ }^{36}$, finden sich auch Notizen über Tätigkeiten, Schicksal oder Charaktereigenschaften verschiedener Persönlichkeiten des 16. Jh. Der frühe Tod des französischen Philologen Estienne Dolet (1508-1546) ging Gessner offenbar nahe, weshalb er dieses Ereignis marginal festhielt. Er notierte, dass Dolet wegen des Druckes eines Neuen Testamentes in französischer Sprache hingerichtet worden sei, womit Dolet für den überzeugten Protestanten Gessner gleichsam zum Märtyrer par excellence wurde. ${ }^{37}$ Märtyrerschicksale trafen Gessner stets innerlich, wovon nicht zuletzt sein warmherziger Trostbrief an verfolgte, von der Todesstrafe bedrohte italienische Protestanten vom Januar 1561 zeugt. ${ }^{38}$ Gessner skizziert an dieser Stelle freilich ein zu idealisiertes Bild des französischen Philologen, zumal dieser in Glaubensfragen eher eigenen Spekulationen als der protestantischen Lehre anhing. ${ }^{39}$

Von Gessners aktiver Auseinandersetzung mit der gesamteuropäischen Gelehrtenwelt zeugen weitere Marginalien verschiedensten Inhaltes. So hielt er etwa fest, dass der französische Philologe und Ichthyologe Pierre Gilles (1490-1555) 1549 Persien bereiste :

«Hoc anno 1549 audivi/ eum in Persia ... peregrinari.» ${ }^{40}$

Zur umstrittenen Konversion des Ingolstädter Philosophieprofessors Veit Amerbach (1503-1557) zum Katholizismus hielt er fest, dass dieser wohl aus selbstsüchtigen Absichten und Geiz diesen Schritt getan habe : 
«Hoc anno ut fertur euan-/ gelice veritati/ renunciavit, et/ ad papistas se contulit, motus ut audio ambitione/ et avaritia. Opinor eum/ iam agere Ingoldstadii.» ${ }^{41}$

Gemäss einer weiteren Notiz gelangte der bekannte Erzbischof von Uppsala, Olaus Magnus (1490-1557), unter Vermittlung des deutschen Arztes Thomas Erastus (1524-1583) mit der Bitte an Gessner, seiner und seines Bruders im Rahmen seiner bibliographischen Arbeiten zu gedenken. Olaus Magnus hatte dabei wohl die von seinem Bruder Johannes Magnus (1488-1544) verfasste und von ihm herausgegebene Historia... de omnibus Gothorum Sueonumque regibus im Auge, die 1554 in Rom erscheinen konnte. Gessner sagte im Dezember 1548 zu, der Bitte zu entsprechen und das Werk in den Appendix Bibliothecae von 1555 aufzunehmen ${ }^{42}$ :

«Commendavit [Olaus Magnus] se mihi/ per Thomam Erastum/ sive Lüberum/ Badensem,/ .../ ut honorificam ipsius et/ fratris mentionem/ facerem. 1548. Decembris./ promisi me/ id facturum/ in Appendice/ ...» ${ }^{43}$

Dieser Passus beweist einmal mehr, in welch hohem Ansehen die Bibliotheca universalis als das massgebliche bibliographische Handwerkszeug des europäischen Gelehrten stand.

Aber nicht nur Geschehnisse und Personen aus dem Ausland, sondern auch seiner geliebten Vaterstadt finden in den Marginalien Erwähnung. Gessner erinnert beispielsweise, dass der italienische Glaubensflüchtling und spätere Antitrinitarier Laelius Socinus (1525-1562) im November 1548 den Zürcher Hebraisten Konrad Pellikan (1478-1556) besuchte:

«Mariani Iunioris Doctoris/ Bononiensis filius, nomine Lelius/ hic fuit apud/ Pellicanum anno/ 1548 Novembri.» ${ }^{44}$

Zwar war bis anhin bekannt, dass Socinus bereits im Herbst 1548 nach Zürich gelangte ${ }^{45}$, jedoch ist Gessners Bemerkung, soweit ich sehen konnte, die einzige Quelle, die seinen Logierort erwähnt.

Eine ähnliche Bemerkung findet sich marginal zum Artikel über den französischen Philologen und Theologen François Vatable (1493-1547). Gessner annotierte, dass Vatable nebst allen aufgeführten Werken auch einen Kommentar zum Propheten Jesaja verfasst habe, welchen ein adliger Pole, wohl handschriftlich ${ }^{46}$, bei sich gehabt habe, als dieser 1549 bei Rudoph Gwalther (1519-1586) in Zürich einkehrte. ${ }^{47}$ Dieser Hinweis verdeutlicht einmal mehr, mit welcher Aufmerksamkeit Gessner alle nur irgendwie greifbaren bibliographischen Informationen sammelte. Wer aber war dieser Pole? Aus den Einträgen zum Jahr 1549 in Heinrich Bullingers Tagebuch geht hervor, dass es sich dabei um Florian Rhozwicz handeln 
muss, der am 29. März 1549 von Warschau kommend Zürich erreichte. ${ }^{48}$ Gessners Bemerkung, dass Rhozwicz bei Gwalther nächtigte, findet ihre Bestätigung in einem Brief Gwalthers an den St. Galler Reformator Vadian vom 21. April 1549, worin er den Polen an Vadian weiterempfahl, nachdem dieser drei Wochen bei ihm gewohnt hatte. ${ }^{49}$ Rhozwicz, besser bekannt unter dem Namen Florian Susliga ${ }^{50}$, war ein Hochstapler und Betrüger. Er hatte sich bei Calvin durch gefälschte Empfehlungsschreiben eingeführt, erschwindelte verschiedenerorts stattliche Geldbeträge und wusste, sich auch bei den Zürchern einzuschleichen. ${ }^{51}$ Es dauerte eine Weile, bis ihm diese auf die Schliche kamen. Obschon Bullinger bereits im Juli 1549 von Laelius Socinus vor Susliga gewarnt worden war ${ }^{52}$, unterhielten die Zürcher mindestens bis zum Herbst gute Beziehungen zu ihm. Auf Susligas Wunsch widmete ihm Gwalther am $1.0 k$ tober 1549 die Komödie Nabal ${ }^{53}$, wozu Gessner ein griechisches Widmungsgedicht verfasste ${ }^{54}$ Beide erhielten sie von Susliga auch einschmeichelnde Büchergeschenke. Gessner sandte er eine Ausgabe der in Hexametern verfassten Weltbeschreibung des Dionysios Periegeta $^{55}$ und Gwalther beehrte er mit den Römischen Altertümern von Dionysios von Halikarnassos. ${ }^{56}$

Ein weiteres Beispiel, welches Gessners Marginalien als historisch wertvolles und interessantes Quellenmaterial evident werden lässt, findet sich in einer Notiz zu einem Autor, dessen Werk Gessner bis 1548 unbekannt war und den er darum handschriftlich in sein Handexemplar einfügte. Es betrifft den aus Bayern stammenden Theologen Paul Rasdorfer (gest. 1564), der auf Empfehlung Bucers an Zwingli in Betschwanden (GL) zum Pfarrer gewählt worden war und der sich stark für die Reformation einsetzte. Im Frühling 1532 musste er Betschwanden verlassen, weilte danach kurz in Zurzach und amtete 1533 bis 1548 als Pfarrer im bayrischen Kempten. Infolge des Interims erneut vertrieben, verbrachte er die Jahre bis zu seinem Tod als Pfarrer im Kanton Bern. ${ }^{57}$ Gessner schrieb :

«Paulus Rassdorpher/ concionator in Kempten/ Germanie civitate,/ ... scripsit/ libellum Germanicum de/ cruce. statim post cladem/ Tigurinam, anno 1532./ (Dz buechli ward verbotten:/ vidi eum Iulio. 1548. Tiguri)»». ${ }^{58}$

Rasdorfer schrieb 1532, kurz nach der vernichtenden Niederlage in der zweiten Schlacht von Kappel, ein Trostbüchlein für die Reformierten mit dem Titel: Crütz mit sinen esten gezwyet durch Paulum Rassdorffer der zyt armen diener des Herren worts in Betschwanden im Land Glaris. ${ }^{59}$ Das Werklein wurde später aus unbekannten Gründen verboten. Vielleicht fühlten 


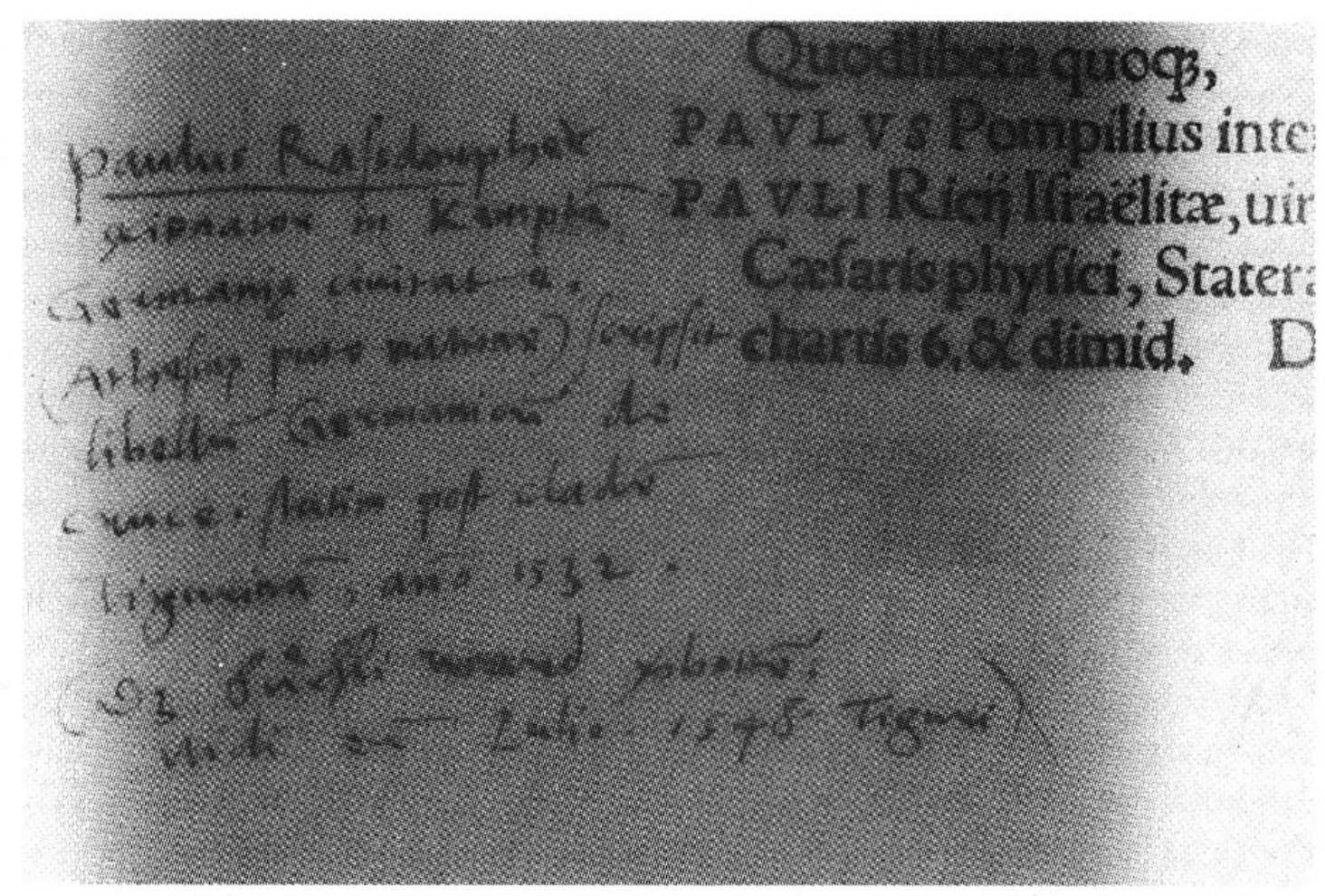

Marginalie von Konrad Gessner im Handexemplar der «Bibliotheca universalis» betreffend Paul Rasdorfer.

sich die katholischen Orte auf irgendeine Art und Weise verletzt oder gar angegriffen und veranlassten den Zürcher Rat, ein entsprechendes Verbot auszusprechen. Denn nach dem Zweiten Kappelerkrieg gestalteten die katholischen Miteidgenossen die Zürcher Zensurgesetzgebung indirekt massgeblich mit, weshalb beispielsweise auch Gwalthers fünf Predigten über den Antichristen von 1546 verboten worden waren. ${ }^{60}$ Gessners Eintrag über die Zensierung von Rasdorfers Werk ist die einzige Mitteilung, die wir über diesen Fall besitzen!

\section{Gessners Beziehungen zu Basler Druckern ${ }^{61}$}

Dass Gessner nicht nur zu Froschauer, sondern auch zu anderen Druckern und Verlegern gute und zum Teil intensive Beziehungen unterhielt ${ }^{62}$, findet seine Bestätigung nicht nur darin, dass er die zwanzig Bücher der 1548 bei Froschauer erschienenen Pandectae, dem Schlagwortkatalog zur Bibliotheca 
universalis, je einem Drucker widmete ${ }^{63}$ oder dass ihm verschiedene Drucker Bücher schenkten ${ }^{64}$, sondern dies kommt auch in den Marginalien zum Handexemplar der Bibliotheca universalis zum Ausdruck. Zum Artikel über Georg Agricola (1494-1555), dem Begründer der Mineralogie und der Bergbaukunde, notierte Gessner an den Rand, dass ihn Hieronymus Froben (1501-1563) im Juni 1548 über gewisse schriftstellerische Vorhaben Agricolas informiert habe:

«Alios/insuper sex libros de rebus/metallicis scribit aut iam/ conscripsit: quibus librum suum/ de ponderibus et mensuris,/ ubi multa contra Alciatum/ scribit, adiunget, ad/ Frobenium missurus, ut/ Frobenius ipse mihi narravit/ 1548. Iunio.» ${ }^{65}$

Gessner interessierte sich demnach besonders für Agricolas Werk über Masse und Gewichte, das einiges gegen das gleichnamige, 1530 in Hagenau erschienene Werk des Andreas Alciatus (1492-1550) vorbringe. Agricola versprach Froben, ihm das Werk nach seiner Fertigstellung zuzusenden, was er offenbar auch tat, denn das Werk konnte 1550 bei Froben erscheinen.

Aus dieser Notiz geht Gessners persönliche Nähe zum ebenfalls zoologisch interessierten ${ }^{66}$ Drucker Hieronymus Froben hervor, dem er im Februar 1549 das 21. Buch der Pandekten De theologia widmete, das im gleichen Jahr als separater Band unter dem Titel Partitiones theologicae erschien. Gessner schrieb in der Widmungsvorrede, dass er Froben und seinem Partner Episcopius ursprünglich das 20. Buch der Pandekten De medicina zueignen wollte. Dies nicht zuletzt daher, weil sie im gleichen Jahr eine Ausgabe der Werke des antiken Mediziners Galen (130-199) zu drucken bereit waren, an der Gessner übrigens auch mitgearbeitet hatte. Da sich der anatomische Unterricht an der Universität Basel damals vornehmlich an Galen orientierte ${ }^{67}$, war dem Werk bereits im voraus ein gewisser lokaler Absatz sicher. Gessner sah sich bekanntlich überfordert, den immensen Stoff für das zwanzigste Buch der Pandekten innert Kürze zu bewältigen, weshalb er ihnen das Buch über die Theologie widmete. Denn auch auf diesem Gebiet hatten sich die beiden Basler Drucker ansehnliche Verdienste erworben. ${ }^{68}$ Das Haus Froben brachte schliesslich 1562 eine weitere Ausgabe der Werke Galens heraus, zu der Gessner eine berühmte Biobibliographie des Griechen verfasste.

Dass Gessner sich von Froben über den Stand der Arbeiten Agricolas ins Bild setzen liess, zeugt von seinem Interesse an den Werken des sächsischen Erdwissenschaftlers ${ }^{69}$, dessen ebenfalls in Basel bei Froben erschienenes Handbuch der Bergbauwissenschaften Bermannus sive de re metallica von 
1530 er besass und das er ebenfalls handschriftlich reich annotierte..$^{70}$ Gessner benutzte Agricolas Arbeiten nicht nur für seine Historia animalium, sondern sehr ausgiebig in seinem einzigen erdwissenschaftlichen Werk $D e$ omni rerum fossilium genere, das 1565 als letztes Werk vor seinem Tod in Zürich erschien und worin Agricola mit Abstand den meistzitierten Autor darstellt. Es ist daher auch kein Zufall, dass die Kurzbiographie über Georg Agricola im von Heinrich Pantaleon bearbeiteten Gelehrtenlexikon der frühen Neuzeit, den 1566 bei Brylinger in Basel gedruckten Prosopographiae heroum atque illustrium virorum totius Germaniae, von Gessner stammt. ${ }^{71}$

Ein weiterer Basler Drucker, dessen Beziehung zu Gessner aufgrund der Marginalien lebendiger und greifbarer wird, ist Johannes Herwagen (14971558), dem Gessner das 14. Buch der Pandekten De naturali philosophia widmete. ${ }^{72}$ Anlass dazu bot Herwagens Ausgabe gewisser Aristoteleskommentare des Tübinger Philosophieprofessors Jakob Schegk (1511-1587) von 1546. Gessner schätzte Schegk als Aristoteleskenner, benutzte gewisse Werke von ihm in seinen Vorlesungen und war ab 1559 mit ihm befreundet. ${ }^{73}$ Zum Artikel über den Augsburger Reformator und späteren Berner Theologieprofessor Wolfgang Musculus (1497-1563) lesen wir die Notiz, dass ihm Herwagen gewisse Teile von dessen Kommentar zum Johannesevangelium geschenkt habe, die Gessner seinerseits seinem Verwandten Kaspar Hirt zur Hochzeit vermacht habe:

«Eiusdem in Ioannem/ Heptades 3. posterio-/ res ii. simul impresse ab/ Hervagio in fol. mihi/ donate sunt, ego eas affi/ ni meo Gaspari/ Hirt in nuptiis cum/ Regula Sprünglerin/ donavi. 1548. Octobri.» ${ }^{74}$

Ein weiterer Eintrag auf der gleichen Seite besagt, dass Musculus seine Übersetzungsarbeiten der griechischen Werke von Prokop im November 1548 abgeschlossen und die Arbeit an Herwagen gesandt habe.

\section{Briefe und Bücher}

Für den Bibliothekar und Historiker von besonderem Interesse sind Gessners marginal angebrachte Bemerkungen über Briefe, die er mit gewissen Gelehrten wechselte, oder über Bücher, die sich in seinem eigenen Besitz oder demjenigen gewisser Freunde und Bekannten befanden. Ein längst bekanntes, aber bedauerlicherweise immer noch unerledigtes Desiderat der Gessnerforschung ist die Erschliessung seines reichen und weit verzweigten Briefwechsels. Für künftige Bearbeiter dieser Korrespondenz sowie für all- 
fällige Biographen dürfte von Interesse sein, dass Gessner in seinen Marginalien auf gewisse Briefe hinweist, die ansonsten verschollen und unauffindbar sind. ${ }^{75}$

Ein erster Brief, den Gessner marginal erwähnt, betrifft eine Anfrage des berühmten französischen Sprachforschers Guillaume Postel (1510-1581) an den Zürcher Hebraisten Theodor Bibliander (gest. 1564). Bibliander und Postel haben sich reichlich Gedanken über die Entstehung der Sprachen und der Sprachenvielfalt gemacht, die Gessner in seinem Werk Mithridates, einem Klassiker der frühen vergleichenden Sprachwissenschaften, reichlich rezipiert hat. Gessner zitiert aus diesem Brief den entsprechenden Passus ${ }^{76}$, in welchem Postel gegenüber Bibliander die Bitte äussert, Gessner Grüsse zu bestellen und ihn zu fragen, ob er, abgesehen von Josephus, Manetho und Tatianus Assyrius, von weiteren antiken Autoren Kenntnis habe, welche über den babylonisch-hellenistischen Geschichtsschreiber Berossus handelten und sich auch über die Glaubwürdigkeit gewisser seiner Schriften äusserten. Was Gessner darauf geantwortet hat, ist ebenfalls unbekannt. Er weist jedoch an anderer Stelle darauf hin, dass er mit Postel auch selber korrespondierte. ${ }^{77}$

Weitere verlorene Briefe, die Gessner anführt ${ }^{78}$, stammen vom Piemonteser Astronomen und Philologen Johannes Ferrerius, der Gessner verschiedentlich Naturalien zukommen liess. ${ }^{79}$ Er sandte ihm 1552 und 1553 je einen Brief, wobei der erste über verschiedene Tiere Schottlands handelte und dem zweiten gewisse Abbildungen derselben beilagen. Gessner erwähnt zudem einen Brief des italienischen Poeten Johannes Franciscus Quintianus Stoa (1486-1557) vom 13. März 1548, dem ein Verzeichnis seiner Schriften beigefügt war ${ }^{80}$, sowie ein Schreiben eines nicht näher bekannten Quintianus medicus aus Italien, der ihm im April 1548 geschrieben habe, dass er sein Werk über De diebus criticis des griechischen Arztes Galen bald herausgeben werde. Ein letzter Brief stammt vom Frankfurter Juristen Justinus Göbler (1503/04-1567), der sich ebenfalls 1548 bei Gessner bedankte, wobei der Dankesgrund verschwiegen wird. ${ }^{81}$

Zu über hundert Werken annotierte Gessner den ihm bekannen Besitzer derselben. Bei 57 Werken vermerkte er, dass er diese selber in seinem Besitz habe, wovon aber heute lediglich noch zwölf auffindbar sind. Ansonsten verweist er auf die Zürcher Fraumünster- und Grossmünsterbibliotheken sowie recht häufig auf die Privatbibliotheken anderer Zürcher Gelehrter wie etwa derjenigen Theodor Biblianders, Heinrich Bullingers, Christoph Clausers, Rudolph Collins, Johannes Fries', Christoph Froschauers, Rudolph 
Gwalthers, Konrad Pellikans, Otto Werdmüllers oder Johannes Wolfs. Darüber hinaus hat Gessner auch nichtzürcherische Privatbibliotheken genauer inspiziert und erinnert sich da und dort an ihre Bestände, so etwa im Fall des Bündner Reformators Johannes Fabritius Montanus (1527-1566) ${ }^{82}$ oder sogar des berühmten katholischen Gegenspielers der Schweizer Reformation, des Glarner Historikers und Staatsmannes Aegidius Tschudi (15051572). Tschudi gehörte seit 1558 zum Patientenkreis Gessners, welchen dieser im gleichen Jahr von einem schwerwiegenden Nierensteinleiden, später aber auch von Fieber und Asthma zu kurieren suchte. ${ }^{83}$ Trotz ihrer konfessionell grundsätzlich gegenteiligen Ansichten stellten diese kein Hindernis im privaten Verkehr zwischen den beiden Gelehrten dar. Offenbar war Gessner selber über diese tolerante Haltung Tschudis ihm gegenüber erstaunt, denn am 28. September 1565 schrieb er an den Augsburger Arzt Adolph Occo (1524-1606), dass Tschudi, obschon er den reformierten Glauben nicht schätze, ihn trotzdem als Arzt in Anspruch genommen habe. ${ }^{84}$ Dass sich die beiden nicht nur über Tschudis Leiden unterhielten, beweisen wiederum zwei Einträge Gessners in seinem Handexemplar der Bibliotheca universalis, in denen er auf gewisse Bücher im Besitz Tschudis zu sprechen kommt. ${ }^{85}$ 


\section{Anmerkungen}

1 Der Autor dieses Beitrages ist gegenwärtig mit der Rekonstruktion der Privatbibliothek Gessners beschäftigt. Ein provisorisches Inventar findet sich in: Leu, Urs B., Conrad Gesner als Theologe - Ein Beitrag zur Zürcher Geistesgeschichte des 16. Jahrhunderts, Diss. phil. I Zürich, Bern, 1990, S. 167-187.

2 Sekundärliteratur zur Bibliotheca universalis z.T. verzeichnet in: Wellisch, Hans H., Conrad Gessner - A Bio-Bibliography, Zug, 1984, S. 121-123.

3 Vgl. Fischer, Hans, Conrad Gessner als Bibliograph, in: Conrad Gessner 1516-1565: Universalgelehrter, Naturforscher, Arzt, Zürich, 1967, S. 32.

4 Zu den Quellen vgl. Leu, Urs Bernhard, wie Anm. 1, S. 108-115.

5 Vgl. Schiess, Traugott, Bullingers Korrespondenz mit den Graubündnern, III.Teil, Oktober 1566 bis Juni 1575, Basel, 1906, S. 524.

6 Dieses Exemplar befindet sich heute in der Kantonsbibliothek Vadiana in St. Gallen und trägt die Signatur: B 30 .

7 Dieses Exemplar befindet sich heute in der Zentralbibliothek Zürich (ZBZ) und trägt die Signatur: IV 02.

8 Vgl. Hieronymus, Frank, Griechischer Geist aus Basler Pressen, Ausstellungskatalog, Publikationen der Universität Basel, Nr. 15, Basel, 1992, S. 682.

9 Wolf, Kaspar, Hyposchesis, sive de Conradi Gesneri stirpium historia... pollicitatio, in: Simmler, Josias, Vita ... Conradi Gesneri, Zürich, 1566, f. 47 r.

10 Vgl. ebd., f. 3 r.

11 Vgl. Milt, Bernhard, Conrad Gessner's «Historia plantarum» (Fragmenta relicta), in: Vierteljahresschrift der Naturforschenden Gesellschaft in Zürich, Jg. 81, 1936, S.289f.

12 Vgl. Merian, Peter, Nachrichten über Felix Platters Naturaliensammlung, in: Bericht über die Verhandlungen der Naturforschenden Gesellschaft in Basel vom August 1838 bis Juli 1840, Heft IV, 1840, S.93-102; Rutsch, R., Originalien der Basler Geologischen Sammlung zu den Autoren des 16.-18. Jahrhunderts, in: Verhandlungen der Naturforschenden Gesellschaft in Basel, Jg. 48, 1937, S. 16-20.

13 Vgl. allg. die einzige Monographie zu Hagenbuch: Ochsner, Johann Jakob, I. Caspar Hagenbuch, in: Neujahrsblatt von der Gesellschaft auf der Chorherrenstube, Zürich, 1826. Hagenbuchs bekannteste wissenschaftliche Leistung ist die Erstpublikation des am 18. Mai 1747 auf dem Lindenhof gefundenen römischen Grabsteines aus dem späten 2.Jh.n. Chr., der den Beleg dafür lieferte, dass Zürich zur Römerzeit nicht, wie lange Zeit angenommen, «Tigurum», sondern «Turicum» geheissen hat. Es handelt sich dabei um den bis heute bedeutendsten römischen Fund in der Stadt Zürich. Vgl. Hagenbuch, Johann Caspar, Tessarakostologion Turicense, sive inscriptio antiqua, ex qua Turici sub imperatoribus romanis stationem quadragesimae galliarum fuisse primum innotescit, Zürich, 1747, in: Hagenbuch, Johann Caspar, Epistolae epigraphicae ..., Zürich, 1747, S. 473-620.

14 Diese Bände befinden sich heute alle in der ZBZ, und die meisten davon in den Signaturen XIX, XX, XXI und XXII, welche die alte Hagenbuch-Bibliothek ausmachen. Ausserdem erwarb Hagenbuch auch zwei Handschriften aus Gessners ehemaligem Besitz. Einerseits einen Manuskriptband mit griechischen Hymnen und Annotationen von Gessners Hand 
(ZBZ: Ms C 296), andererseits autographische Fragmente Gessners zu Xenophon (ZBZ: Ms C 327, S. 193 f.).

15 Hagenbuch, Johann Caspar, Bibliotheca auctorum alphabetica, Bd.5, S.289 (ZBZ: Ms C $319)$.

16 Dieses Exemplar befindet sich heute in der ZBZ unter der Signatur: W 96.

17 Es ist sehr schwierig, Literatur über Gronovius zu finden. Vgl. Jöcher, Christian Gottlieb, Gelehrtenlexikon, Zweiter Band, Hildesheim, Olms-Reprint, 1960, Sp. 1622 f. sowie: Grote Winkler Prins, Encyclopedie in 26 Delen, Amsterdam, 1991, S. 431 f. Vgl. den Briefwechsel Hagenbuch-Gronovius in der ZBZ, enthalten in: Ms C 266-277.

18 Claudius Aelianus, Varia Historia ... curante Abrahamo Gronovio, Leiden etc., 1731 (sechste Seite der «Praefatio»).

19 Vgl. Acta eruditorum anno MDCCXXXI publicata, Leipzig, Bernhard Christoph Breitkopf, 1731, S. 490 .

20 Da die Ehe zwischen Susanna Hagenbuch und Johann Jakob Steinbrüchel kinderlos blieb, vermachte die Witwe die Bücher ihres Mannes der Stadtbibliothek, deren Bestände 1916 in die Zentralbibliothek übergingen. Vgl. den Eintrag in: Schenkungen von Büchern, Manuscripten und Münzen an die Bürger Bibliothek der Stadt Zürich von Anno. 1774, bis 1860, S. 32 (ZBZ: Archiv St 22 b). Vgl. auch: Vögelin, Salomon, Geschichte der Wasserkirche und der Stadtbibliothek in Zürich, Zürich, 1848, S. 114 (auch als Neujahrsblatt der Stadtbibliothek auf das Jahr 1847, 6. Heft).

21 Handschriftlicher Katalog der Hagenbuch-Bibliothek von 1764 : ZBZ, Signatur: Ms C 393.

22 Handschriftlicher Katalog der Hagenbuch-Bibliothek von 1758: ZBZ, Signatur: Ms C 361.

23 Vgl. Catalogus librorum bibliothecae Tigurinae, Bd. 5, Zürich, 1809, S. 300.

24 Vgl. Scherrer-Bylund, Paul, Konrad Gessners verschollenes Handexemplar der Bibliotheca universalis wieder aufgefunden, in: Librarium, Jg. 10, 1967, S. 59-61.

25 Vgl. Gessner, Konrad, Bibliotheca universalis, Zürich, Christoph Froschauer, 1545, f. 268 r. Handexemplar in der ZBZ, Signatur: Dr M 3.

26 Vgl. ebd., f. $464 \mathrm{v}$.

27 Vgl. ebd., f. 219 r.

28 Vgl. ebd., f. $112 \mathrm{v}$.

29 Vgl. Hassinger, Erich, Das Werden des neuzeitlichen Europa 1300-1600, Braunschweig, 1976, S. 232. Braudel, Fernand, Sozialgeschichte des 15.-18. Jh., Bd.2, Der Handel, München, 1986, S. 45.

30 Vgl. Elsas, M. J., Umriss einer Geschichte der Preise und Löhne in Deutschland, Bd. 1, Leiden, 1936, S. 22 ff. sowie ders., ebd., Bd. 2, Teil B, Leiden, 1949, S.6.

31 Vgl. den handschriftlichen Eintrag auf dem Titelblatt in der oberen rechten Ecke von Bullingers Handexemplar der Frobenschen Augustinausgabe, das ebenfalls einen Besitzeintrag aus dem Jahr 1534 aufweist. Die betreffenden Bände gelangten aus noch ungeklärten Gründen in die Bibliothek des Klosters Rheinau und befinden sich heute in der ZBZ unter der Signatur: Rm 73-80.

32 Zur Umrechnung vgl. Hauser, Albert, Vom Essen und Trinken im alten Zürich: Tafelsitten, Kochkunst und Lebenshaltung vom Mittelalter bis in die Neuzeit, Zürich, 1961, S.142f.

33 Vgl. Rosenfeld, Hellmut, Bücherpreis, Antiquariatspreis und Einbandpreis im 16. und 17. Jahrhundert, in: Gutenberg Jahrbuch, Mainz, 1958, S.362. Die ZBZ besitzt einen Oktavband aus dem Besitz Heinrich Bullingers, der im Innendeckel des Einbandes die 
Notiz trägt: 10 Batzen. 1531. (Signatur : Z 224). Da auf dem Titelblatt der Buchpreis mit 1 Pfund und 5 Batzen angegeben ist, kann es sich bei den erwähnten 10 Batzen im Einband daher nicht um den Preis für das Buch, sondern nur um den Preis für das Binden handeln. 10 Batzen wiederum entsprechen ca. eineinviertel Pfund bzw. etwas mehr als einem halben Gulden. Selbstredend kamen die Buchbinderarbeiten für einen Folioband entsprechend etwas teurer.

34 Vgl. die entsprechenden Studentenamtsabrechnungen des Grossmünsterstifts im Staatsarchiv Zürich, Signatur : G II 39.2. Hinzu kamen gewisse Naturalien. Was Gessners Jahresgehalt angeht, sind noch nicht alle Unklarheiten beseitigt. So ist beispielsweise folgende Klage aus dem Jahr 1558 gegenüber Bullinger nicht leicht zu interpretieren : «Wer hätte denn nun mich und die Meinigen erhalten, da $\mathrm{Ihr}$ mir ziemlich lange nicht mehr als 30 Gulden jährlich zukommen liesset?» (zit. nach: Kapp, Friedrich, Geschichte des Deutschen Buchhandels, Leipzig, 1886, S. 314). Ob Gessner hier ein gewisses zusätzliches Arzthonorar seitens der Stadt im Auge hat? Grob gesagt liegen wir sicher nicht falsch, wenn wir davon ausgehen, dass Gessner alles in allem kaum mehr als 120 Gulden bzw. ca. 250 Pfund jährlich von der öffentlichen Hand erhalten hat. Diese Annahme drängt sich auch bei einem Vergleich mit den anderen in G II 39.2 erwähnten Gehältern auf.

35 Gessner nutzte auch die Sitte, dass der Widmungsempfänger einer Widmungsvorrede dies gewöhnlich mit einem Geldgeschenk verdankte. Als Gessner 1551 dem Zürcher Rat den ersten Band seiner «Historia animalium» dedizierte, erhielt er dafür als Gegengabe jährlich 10 Malter Roggen oder Weizen sowie 10 Eimer Wein. Vgl. Kapp, Friedrich, Geschichte des Deutschen Buchhandels bis in das siebzehnte Jahrhundert, Leipzig, 1886, S. 319.

36 Vgl. Gessner, Konrad, wie Anm.25, f.185 r: Zu Conradus Peutingerus schreibt Gessner, dass er im Dezember 1547 gestorben sei, was aber falsch ist. Denn Peutinger starb am 28. Oktober 1547. Zu Paulus Fagius (f. 537v) bemerkt er, dass dieser 1550 verschieden sei, in Tat und Wahrheit starb er im vorhergehenden Jahr.

37 Vgl. Gessner, Konrad, wie Anm. 25, f. 602 v.

38 Vgl. dazu: Leu, Urs B., wie Anm. 1, S. 291.

39 Vgl. Real-Encyklopädie für protestantische Theologie und Kirche, Bd.3, Stuttgart, 1855, S.470. Hassinger, Erich, wie Anm.29, S. 177.

40 Vgl. Gessner, Konrad, wie Anm. 25, f. 549v.

41 Vgl. ebd., f. $626 \mathrm{v}$.

42 Vgl. Gessner, Konrad, Appendix Bibliothecae Conradi Gesneri, Zürich, Christoph Froschauer, 1555, f. 67r.

43 Vgl. Gessner, Konrad, wie Anm.25, f.526v.

44 Vgl. ebd., f. $497 \mathrm{v}$.

45 Vgl. den von Nikolaus Maier am 10.0 ktober 1548 verfassten Brief, der sich wiedergegeben findet in: Sozzini, Lelio, Opere, hsg. von Antonio Rotondo, Florenz, 1986, S. 31 f.

46 Dieses Werk wurde, soweit ich sehen konnte, nie gedruckt und ist daher unbekannt.

47 Vgl. Gessner, Konrad, wie Anm.25, f. 261 r.

48 Vgl. Bullinger, Heinrich, Diarium der Jahre 1504-1574, hsg. v. Emil Egli, Basel, 1904, Reprint: Zürich, 1985, S. 37.

49 Vgl. Arbenz, Emil und Wartmann, Hermann, Vadianische Briefsammlung, Bd.6, 15411551, St. Gallen, 1908, Nr. 1655, S. 793. 
50 Die beste und vollständigste Übersicht zu Susliga bei: Jenny, Beat Rudolf (Hsg.), Die Amerbachkorrespondenz, Band 7, Die Briefe aus den Jahren 1548-1550, Basel, 1973, Nr. 3157, S. 224-230 sowie ders., ebd. Bd.9, Die Briefe aus den Jahren 1553-1555, Berichtigungen und Nachträge zu Bd. 7, Basel, 1982, S. LXIII.

51 Vgl. Wotschke, Theodor, Der Briefwechsel der Schweizer mit den Polen, in: Archiv für Reformationsgeschichte, Texte und Untersuchungen, Ergänzungsband III, Leipzig, 1908, S. 15, Anm. 1 .

52 Vgl. ebd., S.22, Anm. 3.

53 Vgl. ebd., S.27.

54 Die handschriftliche Originalfassung des Gedichtes befindet sich heute in der ZBZ unter der Signatur: Ms A 160.2.

55 Vgl. Wotschke, wie Anm. 51, S. 17.

56 Dieses Exemplar befindet sich heute in der ZBZ unter der Signatur W 84 und trägt die geheuchelte handschriftliche Widmung : «Doctissimo ac praestantissimo viro D. Rodolpho Gualthero/ Tigurino, eiusdem Ecclesiae Doctori. Florianus Susl[iga] a Var-/ schavia. C[anonicus]. V[arsaviensis]. Polonus amoris ergo Dono M[ittit].»

57 Zur Biographie von Rasdorfer vgl.: Zwingli, Huldrych, Sämliche Werke, Bd.9, Briefe, hsg. v. Emil Egli u. a., Leipzig, 1925, S.697; Köhler, Walther, Zu Paul Rasdorfer, in: Zwingliana, Bd.6, Heft 1, 1934, S.59; Bullinger, Heinrich, Briefwechsel, Bd.2, Briefe des Jahres 1532, hsg. v. Ulrich Gäbler u. a., Zürich, 1983, S. 108, Anm. 14.

58 Vgl. Gessner, Konrad, wie Anm.25, f. 539 v.

59 Die ZBZ besitzt zwei Exemplare dieses Werkleins unter den Signaturen : 6.426 a und Ms S 32.3. Vgl. zu diesem Werk allgemein: Heer, Gottfried, Die Gemeinde Betschwanden während der Reformationszeit, Glarus, 1917, S. 27-30.

60 Vgl. Bächtold, Hans Ulrich, Heinrich Bullinger vor dem Rat, Zur Gestaltung und Verwaltung des Zürcher Staatswesens in den Jahren 1531 bis 1575, Diss. phil. I Zürich, Bern, 1982, S. 88103.

61 Vgl. allg. Buess, Heinrich, Conrad Gessners Beziehungen zu Basel, in: Gesnerus, Vol.5, 1948, S.1-29; ders., Conrad Gessner und seine Basler Freunde, in: Basler Jahrbuch 1949, S. 59-69.

62 Vgl. allg. Lutz, Hans, Konrad Gessners Beziehungen zu den Verlegern seiner Zeit, nach seinen Pandekten von 1548, in: Mélanges offerts à Marcel Godet, Neuenburg, 1937, S. 109 117.

63 Vgl. Leu, Urs B., wie Anm. 1, S. 197-201.

64 Der Basler Drucker Nikolaus Brylinger schenkte Gessner beispielsweise eine von ihm selber gedruckte Ausgabe der Werke Xenophons von 1545 (ZBZ, Signatur: II App 5) oder der ebenfalls in Basel tätige Drucker Michael Isengrin eignete Gessner ein kleineres Werk des oströmischen Kaisers Leo VI. zu : Leonis imperatoris de bellico apparatu liber, ..., Basel, Michael Isengrin, 1554 (Besitz: Sächsische Landesbibliothek Dresden).

65 Vgl. Gessner, wie Anm. 25, f. 268 r.

66 Vgl. Buess, Heinrich, Conrad Gessner und seine Basler Freunde, in: Basler Jahrbuch, 1949, S. 65 .

67 Vgl. Hintzsche, E., Anatomische Forschungen im mittelalterlichen Abendland, in: Ciba Zeitschrift, Jg. 8, Nr.96, Basel, Dezember 1944, S. 3437.

68 Vgl. Gessner, Konrad, Partitiones theologicae ..., Zürich, Christoph Froschauer, 1549, f. a2 r. 
69 Vgl. allgemein zu Gessner und Agricola die knappen Bemerkungen von: Epprecht, W., Die Beziehungen zwischen Georg Agricola und Conrad Gessner, in: Vierteljahresschrift der Naturforschenden Gesellschaft in Zürich, Jg. 100, 1955, S.271-276.

70 Dieses Exemplar bildet heute eine der Zimelien der Universitätsbibliothek Basel und steht dort unter der Signatur: Hl. VII. 1.

71 Vgl. Pantaleon, Heinrich, Prosopographiae heroum atque illustrium virorum totius Germaniae, Pars III, Nicolaus Brylingerus, 1566, S. 169. Der Text deckt sich nicht mit demjenigen in der «Bibliotheca universalis».

72 Vgl. Gessner, Konrad, Pandectarum sive Partitionum universalium ... libri XXI, Zürich, Christoph Froschauer, 1548, f. 181 r.

73 Vgl. Hanhart, Johannes, Conrad Gessner-Ein Beytrag zur Geschichte des wissenschaftlichen Strebens und der Glaubensverbesserung im 16ten Jahrhundert, Winterthur, 1824, S. 186.

74 Vgl. Gessner, Konrad, wie Anm. 25, f. 629r.

75 Ich stütze mich dabei wie überhaupt im folgenden auf das von Rudolph Steiger angelegte Verzeichnis der Briefe Konrad Gessners, das sich im sogenannten «Promptuarium Konrad Gessners» in der ZBZ befindet.

76 Vgl. Gessner, Konrad, wie Anm. 25, f. 146r.

77 Vgl. Cordus, Valerius, Annotationes in Pedacij Dioscoridis ... de Medica materia libros V(hsg. von Konrad Gessner), Strassburg, 1561, Vorrede an den Leser, f. b.i (verso).

78 Vgl. Gessner, Konrad, wie Anm. 25, 416v.

79 Vgl. Leu, Urs B., Konrad Gessner und die Neue Welt, in: Gesnerus, Vol.49, 1992, S. 298 f.

80 Vgl. Gessner, Konrad, wie Anm. 25, f.418v.

81 Vgl. ebd., f.474r.

82 Zu Fabritius - Gessner vgl.: Fischer, Hans, Johannes Fabricius Montanus (1527-1566) und Conrad Gessner, in: Vierteljahresschrift der Naturforschenden Gesellschaft in Zürich, Jg. 85, 1940, S.322-328; ders., Ein unveröffentlichter Brief Conrad Gessners (1516-1565) an Johann Fabricius Montanus (1527-1566) in Chur, in: Gesnerus, Vol. 3, 1946, S. 125-130.

83 Vgl. Milt, Bernhard, Gilg Tschudi als Patient von Conrad Gessner, in: Vierteljahresschrift der Naturforschenden Gesellschaft in Zürich, Jg. 95, 1950, S. 58-64. Vgl. auch Gessners Brief an Achilles Pirmin Gasser aus dem Jahr 1563, übersetzt in: Peine, Hans (Hsg.), Briefe Konrad Gesners an seine Freunde Gasser und Culmann, Diss. med., Düsseldorf, 1939, S. 26.

84 Vgl. Wolf, Kaspar (Hsg.), Epistolae medicinales... Conradi Gesneri, Zürich, Christoph Froschauer, 1577, f.78v: «Non amat religionem nostram, me tamen aliquando medico utitur.»

85 Vgl. Gessner, Konrad, wie Anm.25, f.327v. und 569r. Die beiden Einträge betreffen medizinische Werke von Hieronymus Brunschwig und Ponticus Virmius. Beachte : Gessner erwähnt Tschudi auch als nützlichen Zuträger zum 1555 erschienenen Vogelbuch (Historia avium) in: Gessner, Konrad, Historiae animalium liber III, qui est de avium natura, ..., Zürich, Christoph Froschauer, 1555, f. a 6r.

Dr. Urs B. Leu

Zentralbibliothek Zürich

Zähringerplatz 6

8025 Zürich 